\title{
The effects of differing type and magnitude of reward on the contrafreeloading phenomenon in rats
}

\author{
LYNN C. ROBERTSON and SUZANNE C. ANDERSON \\ University of Nevada, Reno, Nevada 89507
}

\begin{abstract}
This investigation was made to determine the effects of the magnitude of reward on contrafreeloading, using food or water as reward. Two quantities were selected for each level of reward quality - a 20-mg-pellet food reward, a 45-mg-pellet food reward, a .01-cc water reward, and a .1-cc water reward. Seven days of training were followed by three test sessions. There was a significantly higher percent of contrafreeloding demonstrated with food as reward than with water and higher number of barpresses with small reward than with large. It was argued that a more appropriate measure should include reference to performance during training. In this approach, contrafreeloading with food and water were virtually the same.
\end{abstract}

It has been observed in experimental studies that animals will continue to make a learned instrumental response to obtain a reward even when the reward substance is freely available. This has been termed contrafreeloading, a term devised from the idea that an organism will "work" rather than "freeload."

Several studies have shown that contrafreeloading has consistently appeared in a variety of conditions when food is the reward. Jensen (1963) found that of the total number of pellets consumed, the percentage an animal obtained by barpressing as opposed to freeloading, increased as the amount of prior operant training increased. Later studies demonstrated the generality of Jensen's findings, and extended the contrafreeloading finding to diverse situations. (Carder \& Berkowitz, 1970; Neuringer, 1969; Singh, 1970; Stolz \& Lott, 1964; Tarte \& Snyder, 1972).

The existence of contrafreeloading across reward qualities, i.e., food vs. water, becomes questionable as a result of both the Carder (1972) and Taylor (1972) studies in which a water-reward group was compared to a food-reward group. Neither Carder nor Taylor obtained contrafreeloading to any great extent when water was the reward.

By using a sucrose solution as reward for food-deprived rats and water as reward for water-deprived rats, Carder (1972) concluded that food, being of higher energy quality, would produce higher activity levels in rats and that this might have accounted for the differences in the degree of contrafreeloading between food and water. He cites Fallon, Thompson, and Schild (1965), who deduced that water is a less potent reinforcer for thirsty rats than is food for hungry

This research was supported by the Department of Psychology, University of Nevada, Reno. Requests for reprints should be sent to the first author, Department of Psychology, University of Nevada, Reno, Nevada 89507. The authors wish to thank W. P. Wallace for his help in instituting this study and for his time and advice. We also wish to extend our gratitude to Michael Pullen, Thomas Turney, and Brent Robertson for their assistance and support. rats. Carder contends that he supported the Fallon et al. hypothesis as a plausible determinant as a result of his finding only $26 \%$ contrafreeloading in the water-reward group as compared to $83 \%$ contrafreeloading in the sucrose-solution reward group.

Taylor (1972) studied the differences in contrafreeloading for water vs. food in a more traditional manner, i.e., using water as reward for water-deprived subjects and food pellets as reward for food-deprived subjects. In contrast to previous studies, Taylor found a very low percentage of contrafreeloading with food as reward (approximately 50\% on the 1st test day), with both rewards decreasing over the 15 test sessions. However, it seems plausible that the different percentage rates between the Carder and Taylor studies were a function of the different reward magnitudes used in the water conditions. Taylor employed a $.01-c c$ dipper, whereas Carder used a .1-cc dipper. Hence, Taylor's subjects had to make 10 times the response to obtain the same amount of water as Carder's subjects made in one response.

Knutson and Carlson (1973), in a study that compared two water groups with two food groups, found that the water-reward groups operantly responded more than the food-reward groups both during training and testing, and that drops in responding from training to testing days were similar for the two types of reward. Knutson and Carlson's study gives credence to the question of magnitude as a variable in contrafreeloading, as their water group $(.01 \mathrm{cc})$ had a higher response rate than the food group ( $45 \mathrm{mg}$ ). These magnitudes were the same as utilized by Taylor (1972), which emphasizes the question of magnitude as a possible explanation in the differences between the water groups in Taylor's and Carder's studies.

It seems that, in the attempts to contrast food and water, one difficulty has been the determination of equivalence in reward magnitude, i.e., it is not clear that the food and water rewards previously used represent 
comparable magnitudes. Thus, the purpose of the present experiment was to examine contrafreeloading with different magnitudes of food and water.

\section{METHOD}

The data will be treated as a 2 by 2 factorial design although, for technical reasons, two separate experiments were run with the water conditions starting before the food conditions.

\section{Subjects}

The subjects were 30 Long-Evans pigmented male rats weighing from 180 to $220 \mathrm{~g}$ upon arrival at the Nevada laboratory approximately 3 weeks before the start of their respective conditions. Subjects were housed in individual cages.

\section{Apparatus}

The experimental chamber was a Lehigh Valley Electronics operant conditioning box mounted in an environmental cubicle from the same company. A speaker was situated within the cubicle and provided white noise at $10 \mathrm{~dB}$. Lehigh Valley electronic equipment was programmed to record each response and to terminate a session automatically after a 15 -min training or testing interval. Cumulative graphs were kept to record the pattern of response activity for each subject. Two magnitudes of rewards were used for each level of reward quality: a .1-cc water dipper which, for descriptive purposes, will be called large water (LW), and .01-cc water reward, small water (SW); a 20-mg J. P. Noyes pellet reward, small food (SF), and a 45-mg J. P. Noyes pellet reward, large food (LF). The water dippers were interchangeable in the solenoid-operated dispenser situated in the center of one of the side walls. A single bar to the right of this mechanism when pressed caused rewards to be delivered on a CRF schedule. Free water was presented via a glass tube extending into the box approximately $7.5 \mathrm{~cm}$ to the left of the liquid dipper on the same wall. A graduated cylinder marked to the nearest $.1 \mathrm{cc}$ was used to measure the amount of free intake after termination of each testing session. For the food conditions, a Lehigh Valley solenoid-operated food dispenser replaced the water feeder in the same location. The free-food dispenser was a tube-like structure attached to the outside of the chamber wall with food pellets dropping down into a small obiong dish attached to the tube with dimensions of approximately $2.5 \times 3.5 \mathrm{~cm}$. Pellets were placed in the tube portion of the dispenser, and as pellets were removed, more pellets would automatically fall into the dish. After each session, pellets from the bottom of the operant box were collected to ensure accurate measurement of intake. The empty free dispensers were present throughout their respective conditions to minimize the novel effect of introducing them on the test day.

\section{Procedure}

Subjects were randomly assigned to conditions with 16 subjects in the two water groups and 14 subjects in the two food groups. The discrepancy in the number of subjects in each group was created by an electrical failure involving two subjects in the food conditions during shaping. Posttraining access to food and water was freely available in the home cage. Subjects in the water groups were maintained on a $231 / 2-\mathrm{h}$ deprivation schedule and were given $15 \mathrm{~min}$ free access to water immediately after participation in each experimental session. Subjects in the food conditions were on a 23 -h deprivation schedule and were given 45 min free access to Purina Lab Chow immediately following participation in each experimental session. The difference in deprivation was due to the relative ease in which subjects could consume required amounts of water as compared to food for maintenance. Food subjects were weighed every other day simply as a check for excessive weight loss. No subject at any time dropped below $80 \%$ of individual weight at the start of training with the mean termination weight at $97 \%$ of starting weight.

All water subjects and all food subjects were randomly sequenced, once before deprivation began, as to the time of day when training or testing would occur. One 15-min session was instituted for the food groups in which pellets were delivered without regard to shaping to ensure that subjects recognized the pellets as a food substance. Subjects were shaped by method of successive approximation for 3 days in 1/2-h sessions per day. On the 4 th day, training sessions of $15 \mathrm{~min}$ began and continued for 7 days, immediately followed by 3 test days in which the reward substance was freely available from the free dispenser in competition with the barpress response. The free noncontingent reward was of the same quality and magnitude as the training reward for each of the food conditions.

\section{RESULTS AND DISCUSSION}

To demonstrate that there was a difference between the four groups over the 7 training days in number of barpresses, an analysis of variance was performed. Figure 1 depicts the results. More responses were made with water, as compared to food reward, $F(1,26)=9.73$, $p<.01$, and with small as compared to large reward, $F(1,26)=134.92, p<.01$. The interaction of the four conditions was also significant, $F(1,26)=18.14, p<.01$, as was the change in barpresses across training days, $F(6,156)=12.68, p<.01$. This significance interacted with food vs. water, $F(6,156)=3.18, p<.01$, as can be seen in Figure 1, where the two water groups fluctuated over days to a greater extent than the two food groups. Further statistics were not performed for training days, as these were only to be used as a reference point for further analysis.

Testing data were approached in three ways, each treated by a separate analysis of variance. First, an analysis by the more predominantly used measurement of percentage of contrafreeloading (C/FL), i.e., operant consumption over the sum of operant and free consumption, revealed a significant difference between food and water in accordance with previous studies, $F(1,26)=6.07, p<.05$. The large and small reward magnitude groups did not differ significantly; however, there was an interaction between the significant effect across trials, $\mathrm{F}(2,52)=6.88, \mathrm{p}<.01$, and magnitude, $F(2,52)=3.53, p<.05$. This interaction can be seen more readily in Figure 2 where the small reward groups declined steadily over the 3 testing days, whereas the large-reward groups decreased and then increased.

It should be pointed out that, although the level of C/FL was significant between food and water, the data did not reveal a large percentage of $\mathrm{C} / \mathrm{FL}$ in relation to most other studies. Perhaps the short 15 -min session affected this, as each subject in the present study was observed to eat or drink the free substance first then proceed to barpress, breaking for only short intervals. Taylor (1972) failed to obtain a large percentage of C/FL with either food or water in a 20 -min session (approximately $50 \%$ on the 1 st test day), while studies 
testing for contrafreeloading with food only have used from $40 \mathrm{~min}$ to $24 \mathrm{~h}$ and have obtained a high percentage of C/FL. It is likely that Carder's (1972) experiment also used less than the minimum 40-min interval in his water condition in view of his 50 barpress criterion. Although he does not report the interval length, according to the present observations of barpressing, whether the magnitude is $.01 \mathrm{cc}$ or $.1 \mathrm{cc}, 50$ barpresses is considerably below the average barpresses emitted by subjects in the present study during a 15 -min period.

The second analysis of variance was performed on the number of barpresses over the 3 testing days. Figure 3 depicts the main effect for large vs. small reward, $F(1,26)=9.02, p<.01$. It is important to note that in comparison to Figure 2, the SW group had more barpress responses than the other three conditions, yet percent of C/FL was very low for this group. As the .01-cc dipper (SW) and the 45-mg pellet (LF) have been the standard sizes used in most previous studies, the data presented in Figures 2 and 3 may explain some of the discrepancies that have been found between food and water and emphasize the need for caution in interpreting studies comparing food with water. Such studies may have an inherent confounding due to response rate differences in training. Figure 3 also shows the significant difference of barpresses across days for the four conditions, $\mathrm{F}(2,52)=7.23, \mathrm{p}<.01$.

The final treatment of the data involved an analysis of variance on a measure of relative performance of barpressing from training to testing days. Degree of freeloading $(F / L)$ for each subject was derived by

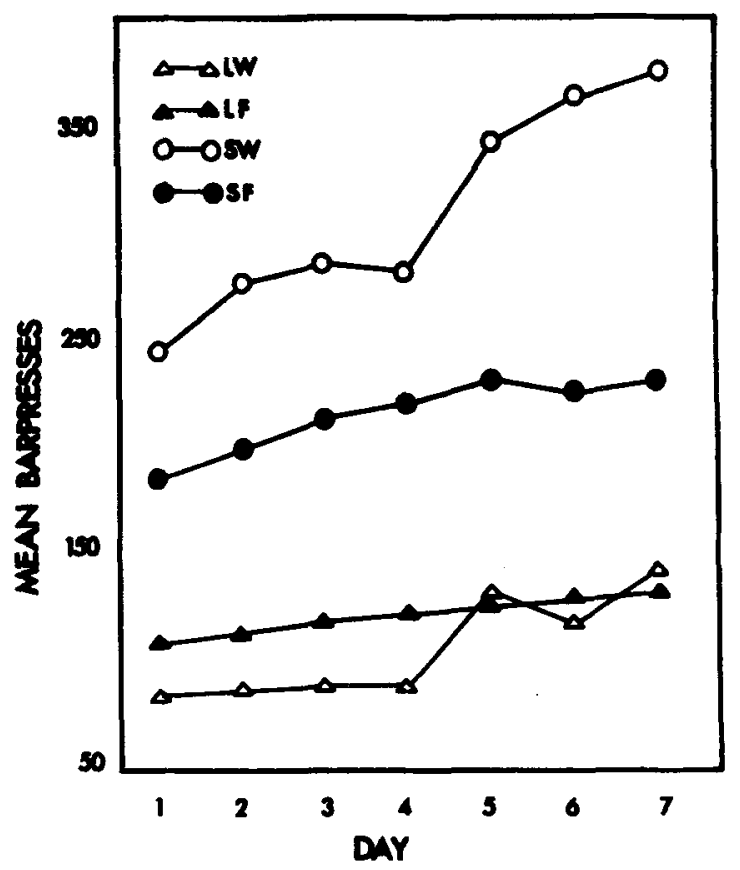

Figure 1. Mean barpresses for the seven training sessions for each of the four experimental conditions.

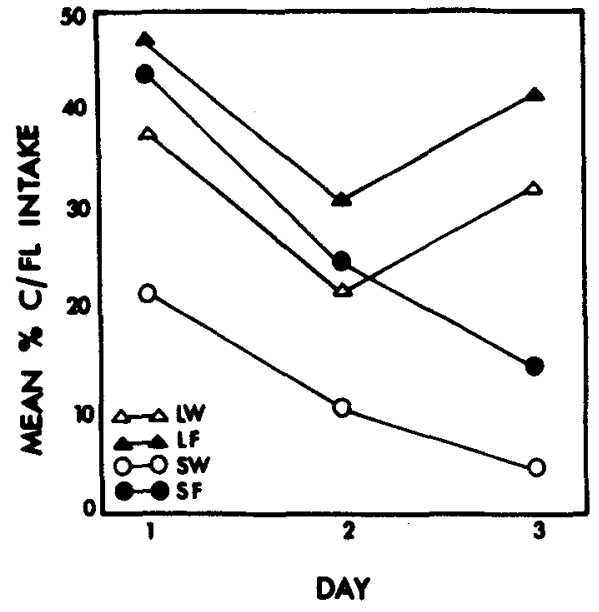

Figure 2. Mean percent of $\mathrm{C} / \mathrm{FL}$ as derived from response intake over the sum of response and free intake for each experimental condition over the 3 testing days.

subtracting number of test barpresses for each of the 3 test days from the number on the last training day and dividing by the latter. For example, a hypothetical subject that barpressed 100 times on the last day of training and 75 times during the first test session would receive a value of .25 , and for 0 barpresses, a value of 1.00. With this method, the actual decrease is given a value rather than the number of test barpresses simply being a percentage of the training performance. This was the only treatment that considered the training days in the analysis, and for this reason it may be a preferred measure. The major disadvantage is that it excludes the amount of noncontingent reward eaten or drunk during testing. However, the fact that barpressing occurs at all in the presence of noncontingent reward is in itself a surprising phenomenon, and the amount of free substance taken from the dish should not be the major concern. To observe simply rate of responding also serves this purpose but does not relate the performance on testing days to any previous performance.

There was a significant effect across trials, $\mathrm{F}(2,52)=17.00, \mathrm{p}<.01$, and trials interacted with reward magnitude, $F(2,52)=7.97, p<.01$. Thus, although magnitude or type of reward did not produce a significant effect on degree of freeloading, magnitude did produce different patterns over the three testing days, a pattern that is depicted in Figure 4.

This effect of degree of $F / L$ seems also to be evident in the Knutson and Carlson (1973) study in which the decreases over food and water appear to be equivalent in their graphs. One puzzling aspect of the Knutson and Carlson study, however, is the reported decline in contrafreeloading within the test session itself, as each subject in the present study showed a distinct within-session pattern of eating or drinking the free substance first and then barpressing as mentioned previously, a pattern apparently opposite to that 


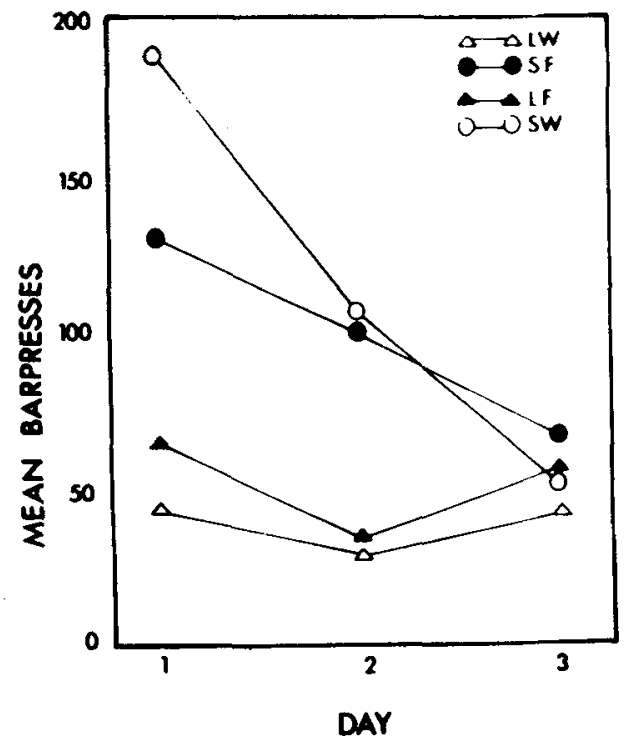

Figure 3. Mean number of barpresses for each of the experimental conditions over 3 testing days.

reported by Knutson and Carlson. Explanations for this discrepancy are not clear. It is possible that procedural variations were responsible for this difference, e.g., Knutson and Carlson allowed two barpresses in two of their conditions before introduction of free food or water, and in the two other conditions allowed eating or drinking from the free substance before insertion of the bar. It appears from the present data that subjects were aware of the noncontingent reward by the observed pattern of eating or drinking before barpressing.

Reward magnitude appears to be an important variable in the study of contrafreeloading, and there seems to be a relationship between the number of

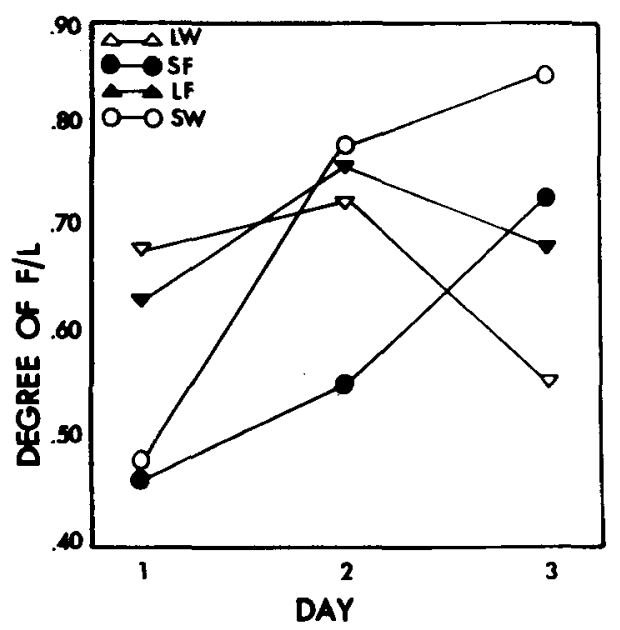

Figure 4. Mean rate of increase and decrease in barpressing response from the last training session in relation to each of three testing sessions for each of the experimental conditions. accumulated responses over training days and the number of operant responses on the critical test day regardless of magnitude or reward. Jensen (1963) also came to this conclusion but only within one reward magnitude. Furthermore, Jensen found an increase of previous responses to affect the ratio of free consumption on test days, whereas the present study suggests a constant ratio between the number of previous responses and the number of testing responses.

In summary, the literature has reported great inconsistencies in the study of contrafreeloading with variations in length of sessions, number of sessions, magnitude of reward, cumulative barpresses before testing, type of reward, analyses of data, etc. The studies imply that this phenomenon does exist, but they are inconclusive as to degree and generality. Any measure may suffice if the only issue is to demonstrate contrafreeloading. That is, responding in the presence of free food may be taken as identifying a somewhat counterintuitive and theoretically intriguing phenomenon. However, if one wants to examine how variables influence contrafreeloading then some care must be exercised. The present study indicates that quantity and quality of reward influence the rate of responding and terminal acquisition levels during training. Only the degree of $F / L$ measure introduced in the present study considers contrafreeloading relative to training performance, and this measure revealed that quantity and quality of reward did not differentially affect contrafreeloading. Thus, the effects demonstrated by the conventional percentage intake measure may reflect, in part or in whole, the influence that these independent variables had on initial training.

\section{REFERENCES}

Carder, B. Rat's preference for earned in comparison with free liquid rein forcers. Psy chonomics Science, 1972, 26, 25-26.

Carder, B., Berkowitz, K. Rat's preference for earned in comparison with free food. Science, 1970, 167, 1273-1274.

Fallon, D., Thompson, D. M., \& Schild, M. E. Concurrent food and water reinforced responding under food, water, and food and water deprivation. Psy chological Reports, 1965, 16, 1305-1311.

Jensen, G. D. Preference for barpressing over "freeloading" as a function of number of rewarded presses. Joumal of Experimental Psychology, 1963, 65, 451-454.

Knutson, John F., \& Carlson, Curtis W. Operant responding with free access to the reinforcer: $A$ replication and extension. Animal Learning and Behavior, 1973, 1, 133-136.

Neuringer, A. J. Animals respond for food in the presence of free food. Science, $1969,166,366-401$.

Singh, D. Preference for barpressing to obtain reward over freeloading in rats and children. Journal of Comparative and Physiological Psychology, 1970, 73, 320-327.

Stolz, S. B., \& Lott, D. F. Establishment in rats of a persistent response producing a net loss of reinforcement. Joumal of Comparative and Physiological Psychology, 1964, 57, 147-149.

Tarte, R. D., \& Snyder, Ron L. Some sources of variation in the barpressing versus freeloading phenomenon in rats. Joumal of Comparative and Physiological Psychology, 1973, B4, 128-133.

Taylor, G. T. A limitation of the contrafreeloading phenomenon. Psychonomic Science, 1972, 29, 173-174.

(Received for publication February 14, 1974; accepted November $20,1974$. 